July 2007

\title{
Soft-photon corrections in multi-body meson decays
}

\author{
GINO ISIDORI \\ INFN, Laboratori Nazionali di Frascati, I-00044 Frascati,Italy
}

\begin{abstract}
The effects due to soft-photon emission (and the related virtual corrections) in multibody decays of $B, D$, and $K$ mesons are analysed. We present analytic expressions for the universal $\mathcal{O}(\alpha)$ correction factors which can be applied to all multi-body decay modes where a tight soft-photon energy cut in the decaying-particle rest-frame is applied. All-order resummations valid in the limit of small and large velocities of the final-state particles are also discussed. The phenomenological implications of these correction factors in the distortion of Dalitz-plot distributions of $K \rightarrow 3 \pi$ decays are briefly analysed.
\end{abstract}

\section{Introduction}

In the last few years the large amount of data collected at flavour factories has allowed to reach statistical accuracies around or below the percent level in several decays modes of $B$, $D$, and $K$ mesons. At this level of accuracy electromagnetic effects cannot be neglected. The theoretical evaluation of these effects is a key ingredient to extract from data a precise information about weak interactions or strong dynamics, such as the determination of CKM matrix elements or the extraction of of $\pi \pi$ scattering lengths.

The theoretical treatment of the infrared singularities generated within QED is a well known subject and one of the pillars of quantum field theory. A clear and very general discussion can be found, for instance, in Ref. [1,2]. These general properties of QED have been exploited in great detail in the case of genuine electroweak processes, or processes which can be fully described within perturbation theory within the Standard Model (SM). More recently, a similar program has been extended to a few decay modes of $K$ and $B$ mesons (see e.g. Ref. [3-7]), which can be described within appropriate effective field theories (EFT). The purpose of the present article is to complement and generalise these EFT studies, analysing the general structure of electromagnetic corrections in multi-particle final states. In particular, we are interested in the distortions of the nonradiative decay distributions (Dalitz plot parameters, form factor slopes, etc...) induced by electromagnetic effects. To a large extent, these effects have a universal (long-distance) 
character: their structure can be evaluated independently of the short-distance dynamics which originate the meson decay.

\section{The photon-inclusive decay distribution at $\mathcal{O}(\alpha)$}

From the experimental point of view, the most convenient infrared-safe observable related to the process $P_{0} \rightarrow P_{1} \ldots P_{N}$ is the differential photon-inclusive distribution

$$
\mathrm{d} \Gamma^{\mathrm{incl}}\left(s_{i j} ; E^{\max }\right)=\left.\mathrm{d} \Gamma\left(P_{0} \rightarrow P_{1} \ldots P_{N}+n \gamma\right)\right|_{\sum E_{\gamma}<E^{\max }},
$$

namely the differential width for the process $P_{0} \rightarrow P_{1} \ldots P_{N}$ accompanied by any number of (undetected) photons, with total missing energy less or equal to $E^{\max }$ in the $P_{0}$ rest frame. In addition to $E^{\max }$, the differential photon-inclusive distribution depends on kinematical variables describing the visible particles. A convenient choice for the latter is 1 ]

$$
s_{i j}= \begin{cases}\left(p_{i}+p_{j}\right)^{2} & i \neq 0, j \neq 0, \\ \left(p_{0}-p_{j}\right)^{2} & i=0, j \neq 0 .\end{cases}
$$

The photon-inclusive distribution in (1) can be decomposed as the product of two theoretical quantities: the so-called non-radiative width, $\mathrm{d} \Gamma^{0}\left(s_{i j}\right)$, which survives in the $\alpha \rightarrow 0$ limit, and the corresponding energy-dependent electromagnetic correction factor $\Omega\left(s_{i j} ; E^{\max }\right)$ :

$$
\mathrm{d} \Gamma^{\text {incl }}\left(s_{i j} ; E^{\max }\right)=\mathrm{d} \Gamma^{0}\left(s_{i j}\right) \times \Omega\left(s_{i j} ; E^{\max }\right) .
$$

At any order in the perturbative expansion in $\alpha$ the energy dependence of $\Omega\left(s_{i j} ; E\right)$ is unambiguous and universal up to terms which vanish in the limit $E \rightarrow 0$ [2]. The $E$ independent part of $\Omega$ contains both universal terms, such as the Coulomb corrections, and non-universal terms depending on the short-distance dynamics which originate the decay. In order to discuss the separation between universal and non-universal terms, we start presenting the calculation of $\Omega\left(s_{i j} ; E\right)$ at $\mathcal{O}(\alpha)$ in the limit of a real point-like effective weak vertex.

The general decomposition of $\Omega\left(s_{i j} ; E\right)$ at $\mathcal{O}(\alpha)$ is

$$
\begin{aligned}
\Omega\left(s_{i j} ; E\right) & =1+\sum_{i, j=0}^{N} Q_{i} Q_{j} J_{i j}\left(s_{i j} ; E\right) \\
J_{i j}\left(s_{i j} ; E\right) & =\frac{\alpha}{\pi}\left[2 b_{i j} \ln \left(\frac{m_{0}}{2 E}\right)+F_{i j}+H_{i j}^{\mathrm{IR}}+H_{i j}^{\mathrm{C}}+H_{i j}^{\mathrm{UV}}+\mathcal{O}(E)\right],
\end{aligned}
$$

where $Q_{i \neq 0}$ are the charges of the final-state particles in units of $e$ and $Q_{0}=-\sum_{i=1}^{N} Q_{i}$. The terms $b_{i j}$ and $F_{i j}$ are unambiguously determined by the real-emission amplitude, while the $H_{i j}$ functions are associated to virtual corrections.

\footnotetext{
${ }^{1}$ Although redundant, this choice of variables allows us to keep the discussion on a general ground.
} 
The $S$ matrix element corresponding to the emission of a real photon can be decomposed as

$$
{ }_{\text {out }}\left\langle P_{1}\left(p_{1}\right) \ldots P_{N}\left(p_{n}\right)+\gamma(\epsilon, k) \mid P_{0}\left(p_{0}\right)\right\rangle_{\text {in }}=-i e \mathcal{M}_{0} \times \hat{K} \times(2 \pi)^{4} \delta^{4}\left(p_{0}-\sum_{i=1}^{N} p_{i}-k\right),
$$

where $\mathcal{M}_{0}$ is the invariant amplitude of the non-radiative process:

$$
{ }_{\text {out }}\left\langle P_{1}\left(p_{1}\right) \ldots P_{N}\left(p_{n}\right) \mid P_{0}\left(p_{0}\right)\right\rangle_{\text {in }}=-i \mathcal{M}_{0}(2 \pi)^{4} \delta^{4}\left(p_{0}-\sum_{i=1}^{N} p_{i}\right)
$$

and

$$
\hat{K}=\sum_{i=0}^{N} Q_{i} \frac{\epsilon \cdot p_{i}}{k \cdot p_{i}}+\mathcal{O}(k) .
$$

The integration of the real-emission amplitude in the soft-photon approximation with a photon-energy cut $E$ (namely neglecting $\mathcal{O}(E)$ terms) and regularizing the infraredsingularities with a photon mass $m_{\gamma}$, leads to

$$
\begin{aligned}
\mathrm{d} \Gamma^{\text {real }}\left(s_{i j} ; E\right) & =\mathrm{d} \Gamma^{0}\left(s_{i j}\right) \times \int_{E_{\gamma}<E} \frac{d^{3} \vec{k}}{(2 \pi)^{3} 2 E_{\gamma}} \sum_{\text {spins }}|\hat{K}|^{2}= \\
& =\mathrm{d} \Gamma^{0}\left(s_{i j}\right) \times \frac{\alpha}{\pi} \sum_{i, j=0}^{N} Q_{i} Q_{j}\left[2 b_{i j} \ln \left(\frac{m_{\gamma}}{2 E}\right)+F_{i j}+\mathcal{O}(E)\right],
\end{aligned}
$$

where $[1,2]$

$$
b_{i i}=\frac{1}{2}, \quad b_{i \neq j}=\frac{1}{4 \beta_{i j}} \ln \left(\frac{1+\beta_{i j}}{1-\beta_{i j}}\right), \quad \beta_{i j}=\left[1-\frac{4 m_{i}^{2} m_{j}^{2}}{\left(s_{i j}-m_{i}^{2}-m_{j}^{2}\right)^{2}}\right]^{1 / 2} .
$$

The finite term $F_{i j}$ depends on the specific cut applied on the (soft) photon energy. Imposing the condition $p_{0} \cdot k<m_{0} E$, corresponding to a cut in the $P_{0}$ rest frame, leads to

$$
F_{i \neq j}=\Delta_{i j} \int_{-1}^{1} \mathrm{~d} z \frac{e(z)}{p(z)\left[e^{2}(z)-p^{2}(z)\right]} \ln \left(\frac{e(z)+p(z)}{e(z)-p(z)}\right),
$$

where

$$
\begin{aligned}
e(z)= & \left(\frac{m_{i} m_{j}}{s_{i j}}\right)^{1 / 2}\left[\gamma_{0 i}(1-z)+\gamma_{0 j}(1+z)\right], & \gamma_{i j}=\frac{1}{\left(1-\beta_{i j}^{2}\right)^{1 / 2}} \\
p(z)= & \left\{\frac{m_{i} m_{j}}{s_{i j}}\left[\left(\gamma_{0 i}^{2}-1\right)(1-z)^{2}+\left(\gamma_{0 j}^{2}-1\right)(1+z)^{2}\right]\right. & \\
& \left.+2\left(\gamma_{0 i} \gamma_{0 j} \frac{m_{i} m_{j}}{s_{i j}}-\Delta_{i j}\right)(1+z)(1-z)\right\}^{1 / 2}, & \Delta_{i j}=\frac{s_{i j}-m_{i}^{2}-m_{j}^{2}}{2 s_{i j}}
\end{aligned}
$$


with the special case $i=j$ given by

$$
F_{i i}=\frac{1}{2 \beta_{0 i}} \ln \left(\frac{1+\beta_{0 i}}{1-\beta_{0 i}}\right), \quad F_{00}=1 .
$$

As far as virtual corrections are concerned, the universal infrared singular term cancels out the $\ln \left(m_{\gamma}\right)$ dependence in Eq. (9), and the remaining finite terms are encoded into the three $H_{i j}$ functions in Eq. (5) . Regularizing UV divergences by means of dimensional regularization and renormalizing the real point-like weak vertex in the $\overline{\mathrm{MS}}$ scheme leads to

$$
\begin{aligned}
H_{i j}^{\mathrm{C}} & =-\frac{\pi^{2}}{2 \beta_{i j}}\left(1-\delta_{i j}\right) \Theta\left(\sqrt{s_{i j}}-m_{i}-m_{j}\right), \\
H_{i j}^{\mathrm{UV}} & =\frac{1}{4} \ln \left(\frac{\mu^{2}}{m_{0}^{2}}\right)\left(-1+3 \delta_{i j}\right), \\
H_{i j}^{\mathrm{IR}} & =\left(1-\delta_{i j}\right)\left\{-\frac{1}{2}+\frac{1}{4} \ln \left(\frac{s_{i j}}{m_{0}^{2}}\right)-\frac{m_{i}^{2}-m_{j}^{2}}{4 s_{i j}} \ln \left(\frac{m_{i}}{m_{j}}\right)+\frac{1}{4} \ln \left(\frac{m_{i} m_{j}}{s_{i j}}\right)\right. \\
- & \frac{1}{4} \beta_{i j} \Delta_{i j} \ln \left(\frac{1+\beta_{i j}}{1-\beta_{i j}}\right)+\frac{1}{4 \beta_{i j}} \ln \left(\frac{s_{i j} \beta_{i j}\left|\Delta_{i j}\right|}{m_{0}^{2}}\right) \ln \left(\frac{1+\beta_{i j}}{1-\beta_{i j}}\right) \\
+ & \left.\frac{1}{8 \beta_{i j}}\left[f\left(\frac{\Delta_{i}-\Delta_{i j} \beta_{i j}}{\Delta_{i}+\Delta_{i j} \beta_{i j}}\right)+2 \ln \left(\frac{s_{i j} \beta_{i j}\left|\Delta_{i j}\right|}{m_{i}^{2}}\right) \ln \left(\frac{\Delta_{i}-\Delta_{i j} \beta_{i j}}{\Delta_{i}+\Delta_{i j} \beta_{i j}}\right)+(i \leftrightarrow j)\right]\right\},
\end{aligned}
$$

where

$$
\Delta_{i}=\frac{s_{i j}+m_{i}^{2}-m_{j}^{2}}{2 s_{i j}}, \quad f(x)=-4 \int_{0}^{x} \mathrm{~d} t \frac{\ln (1-t)}{t}+\ln ^{2}(x) .
$$

The first term, $H_{i j}^{\mathrm{C}}$, which is singular in the limit of vanishing velocity among any pair of charged particles, is a genuine long-distance effect: it corresponds to the Coulomb interaction among the two charged particles. This term can indeed be evaluated also in non-relativistic quantum mechanics by means of semi-classical methods (see e.g. Ref. [8]).

The second term, $H_{i j}^{\mathrm{UV}}$, which depends explicitly on the ultraviolet renormalization scale $\mu$, is manifestly not universal: its scale dependence cancels out in Eq. (3), or in the physical observable, by the corresponding scale dependence of the weak amplitude. The finite $\mathcal{O}(\alpha)$ term resulting after this cancellation cannot be computed without knowing the short-distance behaviour of the amplitude. Note that, in the approximation of a point-like weak vertex, this missing piece affects only the overall normalization of the photon-inclusive distribution and not its kinematical structure.

By construction, $H_{i j}^{\mathrm{IR}}$ is what remains after isolating the manifestly universal and manifestly non-universal terms $H_{i j}^{\mathrm{C}}$ and $H_{i j}^{\mathrm{UV}}$. More explicitly, $H_{i j}^{\mathrm{IR}}$ is the finite part of the universal three-point function function after subtracting ultraviolet and infrared 
divergences and the Coulomb term:

$$
\begin{aligned}
H_{i j}^{\mathrm{IR}}= & 4 \pi^{2}\left(1-\delta_{i j}\right) \Re\left\{\int_{\overline{\mathrm{MS}}} \frac{d^{d} k}{i(2 \pi)^{d}} \frac{\left(2 p_{i}+k\right)_{\mu}\left(2 p_{j}-k\right)^{\mu}}{\left[\left(p_{i}+k\right)^{2}-m_{i}^{2}\right]\left[\left(p_{j}-k\right)^{2}-m_{j}^{2}\right]\left[k^{2}-m_{\gamma}^{2}\right]}\right\} \\
& -H_{i j}^{\mathrm{C}}+\left(1-\delta_{i j}\right)\left[\frac{1}{4} \ln \left(\frac{\mu^{2}}{m_{0}^{2}}\right)+b_{i j} \ln \left(\frac{m_{\gamma}^{2}}{m_{0}^{2}}\right)\right] .
\end{aligned}
$$

\section{Resummations and universal correction factor}

The $E \rightarrow 0$ singular terms in Eq. (5) and the $\beta_{i j} \rightarrow 0$ singular terms in $H_{i j}^{\mathrm{C}}$, which represent the potentially largest correction factors, can be summed to all orders in $\alpha$.

As shown in Ref. [2], the resummation of the $\alpha^{n} \ln ^{n}(E)$ terms allows us to remove the $E \rightarrow 0$ singularity, giving rise to the following exponential term

$$
\Omega_{\mathrm{B}}\left(s_{i j} ; E\right)=\left(\frac{2 E}{m_{0}}\right)^{\frac{2 \alpha}{\pi} B\left(s_{i j}\right)}, \quad B\left(s_{i j}\right)=-\sum_{i, j=0}^{N} Q_{i} Q_{j} b_{i j}>0 .
$$

The resummation of the $\left(\pi \alpha / \beta_{i j}\right)^{n}$ Coulomb terms is encoded by the semi-classical result $[8]$

$$
\Omega_{\mathrm{C}}\left(s_{i j}\right)=\prod_{\{0<i<j\}} \frac{2 \pi \alpha Q_{i} Q_{j}}{\beta_{i j}} \frac{1}{e^{\frac{2 \pi \alpha Q_{i} Q_{j}}{\beta_{i j}}}-1}=1+\frac{\alpha}{\pi} \sum_{i j=0}^{N} Q_{i} Q_{j} H_{i j}^{C}+\mathcal{O}\left(\alpha^{2}\right) .
$$

The $\beta_{i j} \rightarrow 0$ singularity does not disappear and it is strengthened in the case of oppositesign charges (attractive interaction), but it remains an integrable singularity over the final-state phase space.

The two resummed expressions in Eqs. (19) and (20) are relevant in two different kinematical regimes: $\Omega_{\mathrm{C}}\left(s_{i j}\right)$ is relevant in the $\beta_{i j} \rightarrow 0$ limit, while $\Omega_{\mathrm{B}}\left(s_{i j} ; E\right)$ acquires a non-trivial kinematical dependence only in the $\beta_{i j} \rightarrow 1$ limit. We can therefore factorize the two effects up to sub-leading $\mathcal{O}\left(\alpha^{2}\right)$ corrections. This allows us to consider the following generalization for the universal part of the electromagnetic correction factor:

$$
\Omega_{\mathrm{eff}}\left(s_{i j} ; E\right)=\Omega_{\mathrm{B}}\left(s_{i j} ; E\right) \times \Omega_{\mathrm{C}}\left(s_{i j}\right) \times\left[1+\frac{\alpha}{\pi} \sum_{i, j=0}^{N} Q_{i} Q_{j}\left(F_{i j}+H_{i j}^{\mathrm{IR}}\right)\right] .
$$

This expression provides a good description of the leading kinematical corrections induced by soft photons in multi-body meson decay. The approximations/validity-limits of $\Omega_{\mathrm{eff}}\left(s_{i j} ; E\right)$ can be listed as follows:

- The leading kinematical singularities, namely the $\alpha^{n} / \beta_{i j}^{n}$ terms for $\beta_{i j} \rightarrow 0$ and the $\alpha^{n} \ln ^{n}\left(E / m_{0}\right)^{n} \ln ^{n}\left(1-\beta_{i j}\right)$ terms for $\beta_{i j} \rightarrow 1$, are summed to all orders.

${ }^{2}$ The result in Eq. (17) is is valid only for $s_{i j}$ variables in the physical range, namely $s_{i j}$ real and positive (such that all terms in Eq. (17) are real). 
- The regular contribution of the real photon emission $\left(F_{i j}\right)$ is correct up to constant terms of $\mathcal{O}\left(\alpha^{2}\right)$ and energy-dependent terms of $\mathcal{O}(\alpha E / \Lambda)$, where $\Lambda$ is a typical hadronic scale. More precisely, the corrections linear in $E$ are controlled by the derivatives of the non-radiative amplitude with respect the kinematical variables: $\mathcal{O}\left(\alpha E \times \partial \mathcal{A} / \partial s_{i}\right)[9]$. In several cases the tightness on the photon-energy cut necessary to keep these corrections under control can thus be quantitatively controlled by the smoothness of the non-radiative amplitude. In practice, the photon-energy cut is rarely a problem in $\pi$ and $K$ decays 3 , while it is a non-trivial constraint for heavier mesons.

- The virtual corrections encoded in $H_{i j}^{\mathrm{IR}}$ are only the universal contribution of lowenergy photons within an effective theory valid below the scale $\Lambda\left(\Lambda<M_{\rho}\right)$, with real effective couplings in the $\alpha \rightarrow 0$ limit. High-energy modes provides in general additional infrared-safe $\mathcal{O}(\alpha)$ contributions which should be evaluated mode by mode (non-universal terms), and which are different in case of final-state leptons or mesons 4 By an appropriate matching procedure, these additional terms can be reabsorbed into the normalization and the kinematical dependence of the non-radiative amplitude. In light meson $(\pi$ and $K)$ decays these extra terms are necessarily smooth functions of the kinematical variables $\mathcal{O}\left(\alpha s_{i} / \Lambda^{2}\right)$ and thus can be safely neglected. These ultraviolet effects are potentially larger in heavy meson decays, but also in this case they are subleading with respect to the leading logarithmic singularities included in $\Omega_{\mathrm{eff}}\left(s_{i j} ; E\right)$.

- The only cases where virtual effects not included in Eq. (21) are potentially relevant are the singular points corresponding to the formation of Coulomb bound states. A notable example is the pionium formation [10], which has recently been observed in $K \rightarrow 3 \pi$ decays $[11,12]$. Such states are treated here as different final states, which should be eliminated by appropriate kinematical cuts (as done for instance in Ref. [11,12]). Given the extremely narrow widths of Coulomb bound states, and the low probability formation, these effects are relevant only in very tiny regions of the space space and can be safely neglected in heavy-meson decays.

\section{A specific application: $K^{+} \rightarrow \pi^{+} \pi^{+} \pi^{-}$decays}

The high-statistics measurements of the $K \rightarrow 3 \pi$ Dalitz Plot distributions performed by the NA48/2 collaboration $[11,12]$ have recently received a considerable attention because of the possibility to extract a precise information on $\pi \pi$ scattering lengths [13-17].

\footnotetext{
${ }^{3}$ The only exceptions are modes where the bremsstrahlung is strongly suppressed compared to the the direct emission by symmetry arguments, such as the helicity-suppressed $K \rightarrow e \nu(\gamma)$ or the CP-violating $K_{L} \rightarrow \pi^{+} \pi^{-}(\gamma)$.

${ }^{4}$ Having assumed real effective couplings in the $\alpha \rightarrow 0$ limit, we have also ignored the $\mathcal{O}(\alpha)$ electromagnetic corrections to the strong phases of the amplitude. For smooth strong phases these can be easily be incorporated starting from the imaginary part of the three-point function in Eq. (18), as discussed for instance in Ref. [3] for the $K \rightarrow \pi \pi$ case.
} 

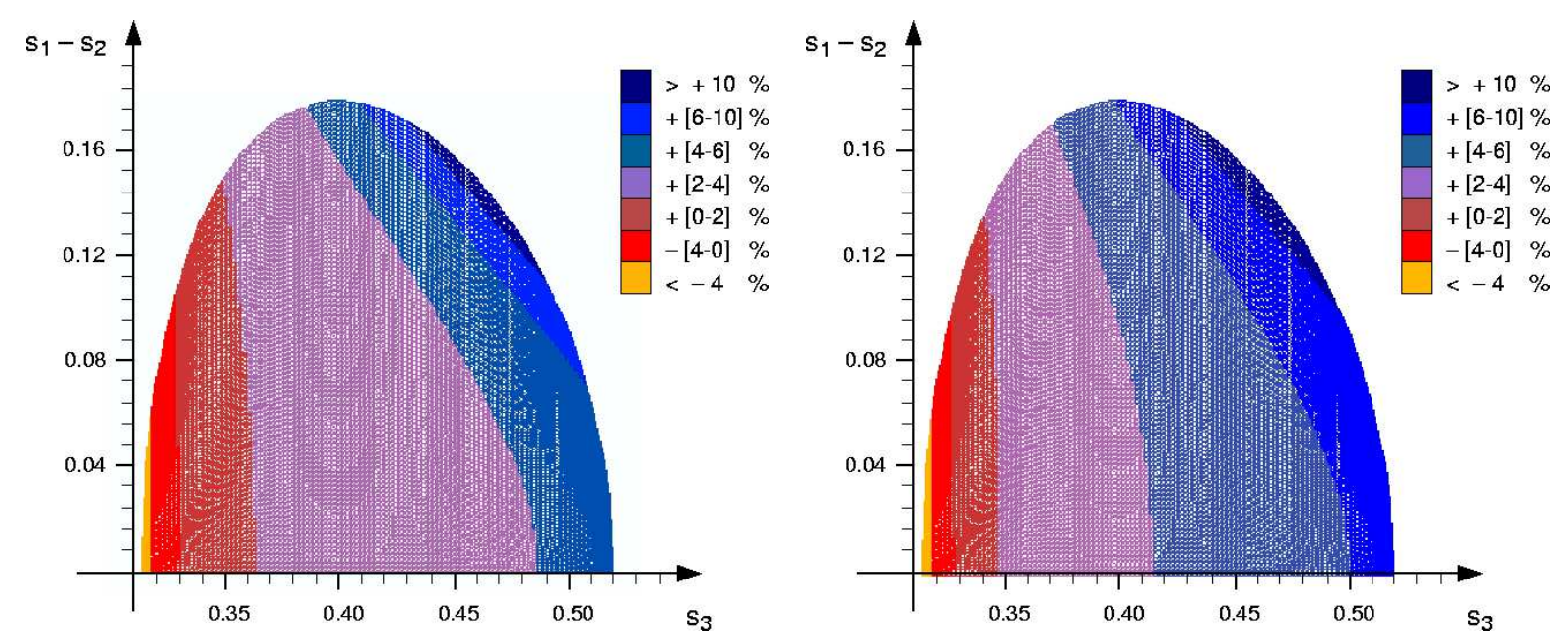

Figure 1: Radiative corrections in the $K^{-} \rightarrow \pi^{+} \pi^{+} \pi^{-}$decay. Left: density plot of $\left[\Omega_{\mathrm{eff}}\left(s_{i j} ; E\right)-1\right]$, evaluated with the full correction term in Eq. (21) with $E=5 \mathrm{MeV}$. Right: density plot of $\left[\Omega_{\mathrm{C}}\left(s_{i j}\right)-1\right]$ (Coulomb term only). The $s_{i}$ are in units of $m_{K}$.

The leading mechanism which allow to measure $\pi \pi$ scattering lengths (and particularly the $a_{0}-a_{2}$ combination) in $K \rightarrow 3 \pi$ decays is the $\pi^{+} \pi^{-} \rightarrow \pi^{0} \pi^{0}$ re-scattering at the $\pi^{+} \pi^{-}$threshold, which produces a prominent cusp in the $M_{\pi^{0} \pi^{0}}$ distribution of the $K^{+} \rightarrow$ $\pi^{+} \pi^{0} \pi^{0}$ decay [13]. The strength of this singularity is proportional to $a_{0}-a_{2}$, but also to phenomenological parameters introduced to describe the $K^{+} \rightarrow \pi^{+} \pi^{+} \pi^{-}$amplitude (see Ref. $[14,15])$. The latter must be determined by experiments from a fit to the $K^{+} \rightarrow \pi^{+} \pi^{+} \pi^{-}$decay distribution, which is likely to receive sizable electromagnetic distortions because of the three charged particles in the final state.

In Fig. 1 we show the impact of soft-photon corrections in the $K^{-} \rightarrow \pi^{+} \pi^{+} \pi^{-}$decay distribution. In particular, we compare the result obtained with the full universal corrections factor in Eq. (21) or using only the Coulomb term in Eq. (20). As expected, radiative corrections induce sizable distortions, especially at the border of the Dalitz plot distribution. However, these are well described by the Coulomb term up an overall normalization factor of $\mathcal{O}(1 \%)$. The procedure adopted by the NA8/2 Collaboration to correct $K^{+} \rightarrow \pi^{+} \pi^{+} \pi^{-}$data using only the Coulomb term is therefore well justified a posteriori.

As discussed in the previous section, our general treatment do note take into account the formation of Coulomb bound states. Such process occur at the border of the $K^{+} \rightarrow \pi^{+} \pi^{+} \pi^{-}$Dalitz plot, when one of the two $\pi^{+} \pi^{-}$pairs is at rest. In order to determine the $K^{+} \rightarrow \pi^{+} \pi^{+} \pi^{-}$decay parameters relevant to the analysis of Ref. [14,15], the narrow regions at the border of the Dalitz plot with Coulomb corrections of $\mathcal{O}(100 \%)$ should therefore be eliminated by appropriate kinematical cuts. This procedure is perfectly consistent with the cut of the pionium region (around the peak of the $M_{\pi^{0} \pi^{0}} \operatorname{cusp}$ ) performed in Ref. [11]. 


\section{Acknowledgments}

We thank Italo Mannelli for interesting discussions which initiated this analysis. This

work is supported in part by the EU Contract No. MRTN-CT-2006-035482 FLAVIAnet.

\section{References}

[1] D. R. Yennie, S. C. Frautschi and H. Suura, Annals Phys. 13 (1961) 379.

[2] S. Weinberg, Phys. Rev. 140 (1965) B516.

[3] V. Cirigliano, J. F. Donoghue and E. Golowich, Eur. Phys. J. C 18 (2000) 83 hep-ph/0008290.

[4] V. Cirigliano, H. Neufeld and H. Pichl, Eur. Phys. J. C 35 (2004) 53 hep-ph/0401173;

V. Cirigliano et al. Eur. Phys. J. C 23 (2002) 121 hep-ph/0110153.

[5] J. Bijnens and F. Borg, Eur. Phys. J. C 39 (2005) 347 hep-ph/0410333; ibid. 40 (2005) 383 hep-ph/0501163.

[6] C. Gatti, Eur. Phys. J. C 45 (2006) 417 hep-ph/0507280;

T. C. Andre, hep-ph/0406006.

[7] E. Baracchini and G. Isidori, Phys. Lett. B 633 (2006) 309 hep-ph/0508071].

[8] See e.g. L.I. Schiff, Quantum Mechanics (McGraw Hill, 1968).

[9] G. D'Ambrosio, G. Ecker, G. Isidori and H. Neufeld, Phys. Lett. B 380 (1996) 165 hep-ph/9603345.

[10] Z. K. Silagadze, JETP Lett. 60 (1994) 689 hep-ph/9411382.

[11] J. R. Batley et al. [NA48/2 Collaboration], Phys. Lett. B 633 (2006) 173 hep-ex/0511056.

[12] E. Goudzovski [NA48/2 Collaboration], arXiv:0706.4059 [hep-ex].

[13] N. Cabibbo, Phys. Rev. Lett. 93 (2004) 121801 hep-ph/0405001.

[14] N. Cabibbo and G. Isidori, JHEP 0503, 021 (2005) hep-ph/0502130.

[15] G. Colangelo, J. Gasser, B. Kubis and A. Rusetsky, Phys. Lett. B 638 (2006) 187 hep-ph/0604084.

[16] E. Gamiz, J. Prades and I. Scimemi, Eur. Phys. J. C 50 (2007) 405 hep-ph/0602023.

[17] S. R. Gevorkyan, D. T. Madigozhin, A. V. Tarasov and O. O. Voskresenskaya, hep-ph/0702154. 